TITLE:

\title{
Revisiting the role of magnetic field fluctuations in nonadiabatic acceleration of ions during dipolarization
}

$\operatorname{AUTHOR}(\mathrm{S}):$

Nosé, M.; Ono, Y.; Christon, S. P.; Lui, A. T. Y.

\section{CITATION:}

Nosé, M.... [et al]. Revisiting the role of magnetic field fluctuations in nonadiabatic acceleration of ions during dipolarization. Journal of Geophysical Research 2012, 117: A02207.

ISSUE DATE:

2012-02

URL:

http://hdl.handle.net/2433/154859

\section{RIGHT:}

(C)2012. American Geophysical Union.; This is not the published version. Please cite only the published version.; この論文は出版社版でありませ ん。引用の際には出版社版をご確認ご利用ください。 


\title{
Revisiting the role of magnetic field fluctuations in nonadiabatic acceleration of ions during dipolarization
}

\author{
M. Nosé, ${ }^{1}$ Y. Ono, ${ }^{2,3}$ S. P. Christon, ${ }^{4}$ and A. T. Y. Lui ${ }^{5}$
}

\begin{abstract}
Using energetic (9-212 keV/e) ion flu data obtained by the Geotail spacecraft, Ono et al. (2009) statistically examined changes in the energy density of $\mathrm{H}^{+}$and $\mathrm{O}^{+}$ions in the near-Earth plasma sheet during substorm-associated dipolarization. They found that ions are nonadiabatically accelerated by the electric fiel induced by the magnetic fiel fluctuation whose frequencies are close to their gyrofrequencies. The present paper revisits this result and find it still holds.
\end{abstract}

\section{Introduction}

Using 10 years of energetic (9-212 keV/e) ion data obtained by the suprathermal ion composition spectrometer (STICS) sensor of the energetic particles and ion composition (EPIC) instrument on board the Geotail spacecraft, Ono et al. [2009] statistically examined changes in the energy density of $\mathrm{H}^{+}$and $\mathrm{O}^{+}$ ions in the near-Earth plasma sheet $\left(X=-16 R_{E}\right.$ to $\left.-6 R_{E}\right)$ during substorm-associated dipolarization. For more than 50 events of dipolarization, Ono et al. [2009] plotted the $k$ ratio (i.e., the ratio of the spectral index before and after dipolarization) as a function of $\chi\left(=\tau_{B} / \tau_{C}\right.$, i.e., the dipolarization time scale divided by the gyroperiod of ions) for both $\mathrm{H}^{+}$and $\mathrm{O}^{+}$ions (see Figure 7 in Ono et al. [2009]). In this, following was observed: (1) whether $\chi$ is close to 1 or not, almost all events are distributed above the $k$ ratio line of 1 for both $\mathrm{H}^{+}$and $\mathrm{O}^{+}$ions and (2) there is no tendency for the $k$ ratio to increase as $\chi$ approaches 1 . These results led Ono et al. [2009] to conclude that the magnetic fiel variations over the time scale of dipolarization is unlikely to be essential for the acceleration of ions. Meanwhile, an objection was recently made to the above conclusion. In the objection, the frequency distribution of events in the $k$ ratio was examined by using data from Figure 7 in Ono et al. [2009] (Figure 1a). It was argued that the frequency distribution for $\mathrm{O}^{+}$ions shifts towards larger $k$ ratio values than the frequency distribution for $\mathrm{H}^{+}$ions. This provides evidence for ion acceleration by a magnetic fiel change on the time scale of dipolarization, which is the viewpoint developed in Delcourt et al. [1990, 1997].

\section{Analysis and Result}

The focal point of this rebuttal is whether the sentence in the objection, "It is clearly apparent ... that the $\mathrm{O}^{+}$profil ... spreads towards $k$ ratios larger than that those of $\mathrm{H}^{+} \ldots$. is correct or not. In this addendum, the statistical significanc of this sentence is examined. First, we calculate the mean value and its standard error of the $k$ ratio for $\mathrm{H}^{+}$and $\mathrm{O}^{+}$ions. Results are $\log _{10}(k$ ratio $)=0.197 \pm 0.026$ for $\mathrm{H}^{+}$ions and $\log _{10}(k$

\footnotetext{
${ }^{1}$ Data Analysis Center for Geomagnetism and Space Magnetism, Graduate School of Science, Kyoto University, Kyoto, Japan.

${ }^{2}$ Graduate School of Science, Kyoto University, Kyoto, Japan.

${ }^{3}$ Now at Information and Media Processing Laboratories, NEC Corporation, Kanagawa, Japan.

${ }^{4}$ Focused Analysis and Research, Columbia, Maryland, USA.

${ }^{5}$ Johns Hopkins University Applied Physics Laboratory, Laurel, Maryland, USA
} 
ratio) $=0.234 \pm 0.035$ for $\mathrm{O}^{+}$ions, which are plotted in the top part of Figure 1a. The ranges of the mean value largely overlap each other, statistically indicating that there is no difference in the mean values of the $k$ ratio between $\mathrm{H}^{+}$and $\mathrm{O}^{+}$. Thus, we believe that the current statistical analysis does not support the objection. Second, we examine which ion has a larger number of events in each bin of the $k$ ratio. As shown in Figure $1 \mathrm{~b}$, this is based on the idea that if the frequency distribution is Gaussian and the distribution for $\mathrm{O}^{+}$is shifted towards higher values of the $k$ ratio than that for $\mathrm{H}^{+}, \mathrm{O}^{+}$will have a larger number of events than $\mathrm{H}^{+}$in each bin when the $k$ ratio is larger than its mean value. Thus it is expected that the number of bins of $n\left(\mathrm{H}^{+}\right)<n\left(\mathrm{O}^{+}\right)$is larger than that of $n\left(\mathrm{H}^{+}\right)>n\left(\mathrm{O}^{+}\right)$, where $n\left(\mathrm{i}^{+}\right)$denotes the number of events in each bin for the ion species $\mathrm{i}^{+}$. On the other hand, in the range of the $k$ ratio where it is smaller than its mean value, $\mathrm{H}^{+}$will have a larger number of events than $\mathrm{O}^{+}$in each bin. Similarly, the number of bins of $n\left(\mathrm{H}^{+}\right)>n\left(\mathrm{O}^{+}\right)$is anticipated to be larger than that of $n\left(\mathrm{H}^{+}\right)<n\left(\mathrm{O}^{+}\right)$. In Figure 1a, we take the mean value of $\log _{10}(k$ ratio) as $0.2155(=(0.197+0.234) / 2)$ and count the number of bins to complete Table 1 . When the $k$ ratio is larger than its mean value, the result in Table 1 is consistent with what is expected from the frequency distribution for $\mathrm{O}^{+}$shifting towards higher values than that for $\mathrm{H}^{+}$. However, when the $k$ ratio is smaller than its mean value, this is not the case. The objection is therefore not strongly supported by this analysis.

\section{Summary}

In summary, we consider that the assertion of the objection is not statistically justifie from Figure 1a and the conclusion of Ono et al. [2009] still holds. However, it should be noted that this addendum does not completely rule out the possibility of the acceleration of ions by magnetotail dipolarization. This acceleration process is numerically well confirme by Delcourt et al. [1990, 1997] and also examined in Figure 6 of Ono et al. [2009]. In some events where the time scale of the magnetic fiel reconfiguratio is comparable to the gyroperiod of ions, in particular, for $\mathrm{O}^{+}$ions, this acceleration process becomes important. Future studies will include a numerical investigation of the relative importance of ion acceleration between the magnetic fiel change on the time scale of dipolarization and the magnetic fiel fluctuation in a shorter time scale causing quasi-resonance with ions.

\section{Acknowledgements}

We are thankful to S. Machida for his helpful comments. This work was supported by the Ministry of Education, Science, Sports and Culture, Grant-in-Aid for Young Scientists (B) (grant 22740322) and Grant-in-Aid for Scientifi Research (B) (grant 22340146). This paper was originally drafted as a Reply to a Comment. The Comment was withdrawn, but the contents of the Reply were judged to be helpful to the readers of the Journal. So, the Editor arranged for this paper to be published as a Brief Report.

Masaki Fujimoto thanks the reviewer for his or her assistance in evaluating the paper.

\section{References}

Delcourt, D. C., A. Pedersen, and J. A. Sauvaud (1990), Dynamics of single-particle orbits during substorm expansion phase, J. Geophys. Res., 95, 20,853-20,865, doi:10.1029/JA095iA12p20853.

Delcourt, D. C., J.-A. Sauvaud, and T. E. Moore (1997), Phase bunching during substorm dipolarization, J. Geophys. Res., 102, 24,31324,324, doi:10.1029/97JA02039.

Ono, Y., M. Nosé, S. P. Christon, and A. T. Y. Lui (2009), The 
role of magnetic fiel fluctuation in nonadiabatic acceleration of ions during dipolarization, J. Geophys. Res., 114, A05209, doi: 10.1029/2008JA013918. 
Figure 1. (a) The frequency distribution of events in the $k$ ratio for $\mathrm{H}^{+}$ions (blue line) and $\mathrm{O}^{+}$ions (red line). The original data taken from Figure 7 of Ono et al. [2009] are used. In the top part, the mean value and its standard error (SE) of the $k$ ratio are displayed with a triangle and a horizontal bar, respectively. (b) Examples of the Gaussian frequency distributions, which shift towards higher/lower values of the $k$ ratio for $\mathrm{O}^{+} / \mathrm{H}^{+}$ions.

Table 1. Number of bins that satisfy conditions regarding the $k$ ratio and comparison of the number of events between $\mathrm{H}^{+}$and $\mathrm{O}^{+} . n\left(\mathrm{i}^{+}\right)$denotes the number of events in each bin for the ion species $\mathrm{i}^{+}$

\begin{tabular}{lccc}
\hline & $n\left(\mathrm{H}^{+}\right)>n\left(\mathrm{O}^{+}\right)$ & $n\left(\mathrm{H}^{+}\right)=n\left(\mathrm{O}^{+}\right)$ & $n\left(\mathrm{H}^{+}\right)<n\left(\mathrm{O}^{+}\right)$ \\
\hline $\log _{10}(k$ ratio $) \geq 0.2155$ & 2 & 0 & 4 \\
$\log _{10}(k$ ratio $)<0.2155$ & 2 & 2 & 2 \\
\hline
\end{tabular}


(a)
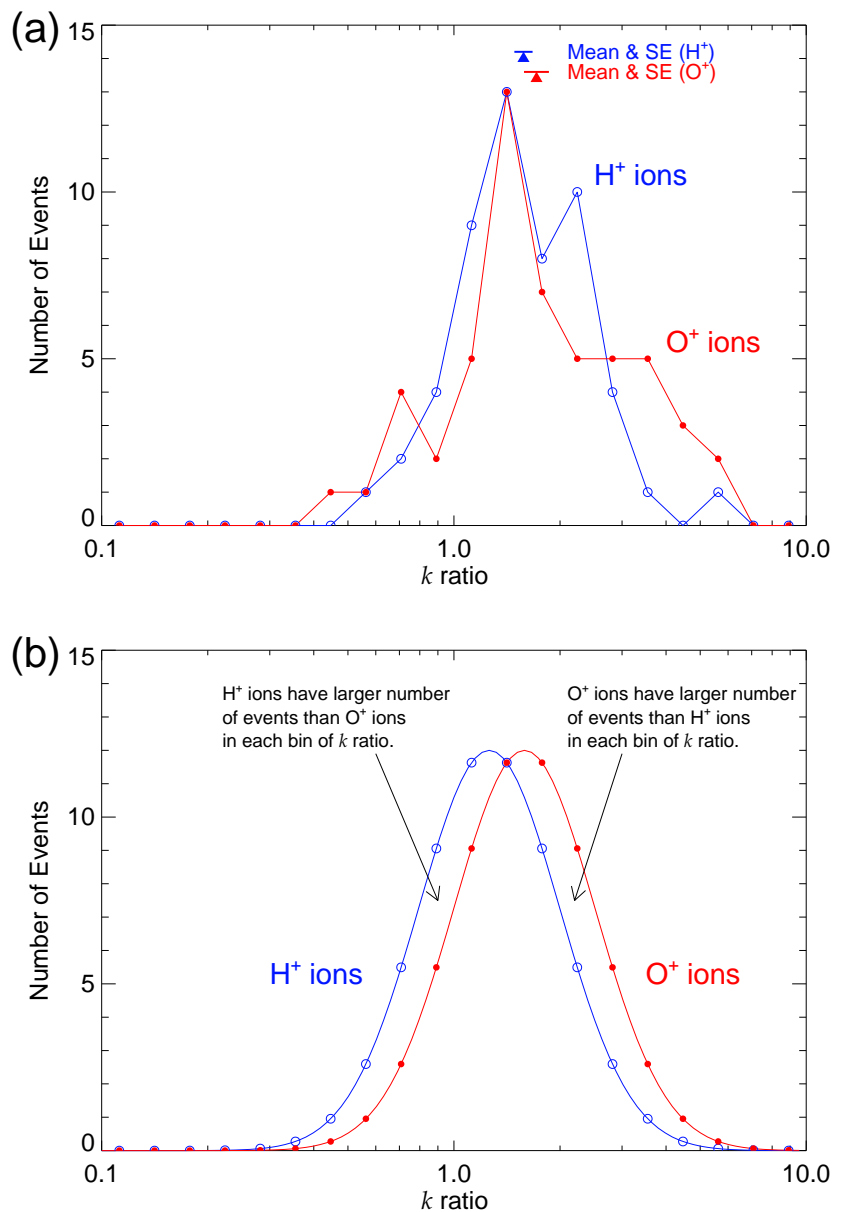